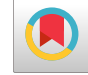

\title{
Psychometric Properties of the Persian Version of Children's Sleep Comic
}

\author{
Mozhgan Valipour ${ }^{1}$, Minoo Kalantari (iD ${ }^{1,{ }^{*}}$ and Seyed Mehdi Tabatabaee (iD ${ }^{1}$ \\ ${ }^{1}$ School of Rehabilitation, Shahid Beheshti University of Medical Sciences, Tehran, Iran \\ "Corresponding author: School of Rehabilitation, Shahid Beheshti University of Medical Sciences, Tehran, Iran. Email: mn_kalantari@yahoo.com
}

Received 2020 February 15; Revised 2020 June 23; Accepted 2020 August 19.

\begin{abstract}
Background: Insomnia is one of the most common sleep problems among children and is associated with a wide variety of impairments such as aggression, decreased attention and concentration, daytime sleepiness, hyperactivity, and irritability. A child with insomnia can affect the whole family. It is important to assess insomnia and sleep behaviors of children because insomnia is not a common diagnosis in them. Children's Sleep Comic is a questionnaire to diagnose insomnia in children aged 5-11 years, which can be completed within 20 minutes.

Objectives: The aim of the present study was to translate and investigate the Persian form of Children's Sleep Comic's content validity and reliability.

Methods: This cross-sectional study was carried out on 209 children aged 5-11 years in primary schools and preschools of Tehran who were selected by random cluster sampling in 2019. The translation was done with the forward-backward method. The content validity was evaluated by 10 occupational therapists, and quantitative content validity was determined by calculating the Content Validity Index (CVI) and Content Validity Ratio (CVR). Internal consistency was assessed through Cronbach's alpha and test-retest reliability via Intraclass Correlation Coefficient (ICC) among 30 children after one week as well as Standard Error of Measurement (SEM).

Results: Overall, 220 children were entered into the study; however, 11 children were excluded because of missing data. The translation of Children's Sleep Comic was considered easy and acceptable. The content validity, which was assessed by CVR and CVI were desirable (0.8 and 0.8, respectively), and the internal consistency was 0.76. ICC and SEM were 0.97 and 0.02, respectively.

Conclusions: The findings of this study showed that the Persian version of the Children's Sleep Comic questionnaire is a reliable and valid instrument that can be used for screening insomnia in Iranian children aged 5-11 years.
\end{abstract}

Keywords: Insomnia, Children, Sleep Disorders, Validation

\section{Background}

Approximately one-third of human life is devoted to sleeping. Sufficient sleep is indispensable for normal brain function. Since sleep aids in lessening anxiety and stress and contributes to the recuperation of energy, it not only increases concentration and consistency of senses but also adds pleasure and joy to activities of daily living. Therefore, we should not consider sleeping a waste of time (1-3). Children frequently do not get sufficient sleep on a regular basis. Getting good sleep is as significant as daily exercise and getting suitable nourishment. Sleep can be considered "nutrition for the brain". Insufficient sleep can disrupt neurological and physical capabilities, diminishing the level of concentration and leading to poor academic performance that can be linked to cognitive, behavioral, and emotional problems being misdiagnosed with disor- ders (4-8). In addition, insufficient sleep may reduce reaction time and increase the risk of accidents (9). Parents commonly estimate that $50 \%$ of their infants, $30 \%$ of children, and $40 \%$ of teenagers have difficulties with sleeping. Self-report data has been consistent with this estimation so that one study found that $60 \%$ of fifth-grade students reported sleep problems unknown to their parents. Despite the high rate of sleep problems in the pediatric population, they remain largely undiagnosed (10). considering the high prevalence of childhood insomnia and the severe consequences associated with it, questionnaires are indeed beneficial in recognition of sleep problems at a young age and an early stage (11).

To the best of our knowledge, there is no accredited scale to evaluate insomnia among Iranian children aged 511 years. 


\section{Objectives}

The objective of this study was to validate this questionnaire and report its psychometric features for research and clinical objectives in Iran.

\section{Methods}

\subsection{Children's Sleep Comic Questionnaire}

The Children's Sleep Comic, designed by Barbara Schwerdtle in 2012 and revised in 2016, is a self-report questionnaire that is standardized in Germany for screening insomnia in children aged 5 to 11 years. This questionnaire contains 20 items accompanied by various answer options about sleep problems and sleep, containing sleep hygiene, sleep quality, duration of sleep, anxiety related to sleep, nightmares, sleepwalking and early morning awakening, napping, and impairments during the day. A cartoon is drawn for every item or an explanation is given for each response to which a statement is allocated. In addition, a comic book questionnaire enables younger children to respond to the questions beside an interviewer even without being literate. Two impartial groups of specialists in childhood sleep disorders categorized the response options as positively affecting sleep, disturbing sleep, or having no effect. Considering the number of responses that belong to negatively affecting sleep options, a number of items were added to obtain an Intensity of Sleep Problem Score (ISPS), which allowed for quantitative analysis. The maximum ISPS score is 20 , and higher scores indicate severe sleep disturbance (12). In this study, we used the revised version of this questionnaire, which is introduced by Schwerdtle in 2016.

\subsection{Translation}

In the beginning, the consent for interpretation and translation of the questionnaire in Iran was acquired from the developer of the instrument. To translate the questionnaire from English to Persian, a standard method used, including forward-backward translation as well as pre-exam by two bilingual people other than primary translators to be impeccably transformative into each other from viewpoints of conceptual and linguistic.

\subsection{Participants}

According to validity and reliability guidelines, 11 samples were required for each item in the present study, so 220 samples were selected through randomized cluster sampling (13). With the permission of the Ministry of Education, a list of public primary schools in Tehran was prepared, of which ten schools (five boys 'schools and five girls' schools) were randomly selected.
Of each school, a class was randomly selected, and their eligibility was assessed. The inclusion criteria in the study were the absence of severe stress over the previous twelve months (for example, due to the death of an immediate family member or migration), not consuming medication that could affect sleeping, and not suffering from an illness requiring hospitalization or long-term and critical treatment based on the report of parents and health record of students. To collect the data, the researcher arranged a session to meet the parents of the chosen children in coordination with the headmaster of the school. Informed consent forms were given to them, and the purpose of the study was clearly described. In total, 220 questionnaires were distributed among children, of which 209 questionnaires were completed, and 11 were excluded due to missing data. Children aged 8-11 responded to the Children's Sleep Comic independently. The examiner was there to answer the child's questions. Children aged 5-7 were guided by an examiner to complete the form. The examiner read and explained the questions and answered to the child with pictures.

The code of ethics was obtained from the Ethics Committee of the Shahid Beheshti University of Medical Sciences (IR.SBMU.RETECH.REC.1398.393).

\subsection{Reliability}

The internal consistency of the questionnaire was assessed with Cronbach's alpha coefficient. To examine testretest reliability, we assessed the Standard Error of Measurement (SEM), and the questionnaires were twice distributed among 30 children after one week. The results were assessed by the Intraclass Correlation Coefficient (ICC).

\subsection{Validity}

In this study, the Content Validity Ratio (CVR) and Content Validity Index (CVI) were calculated. Content validity was done with 10 experts who evaluated the questionnaire based on four criteria of relevancy, clarity, simplicity, and necessity. To calculate the CVR, the necessity of each question (NE) was determined by the formula [CVR $=(\mathrm{NE}-\mathrm{N} / 2)$ / $(\mathrm{N} / 2)$ ]. According to Lawsh's table, if 10 specialists completed the form, the minimum CVR for each item should be 0.62 (14). Waltz and Bausell's method was used to determine the CVI. Experts were asked to evaluate the relevancy, clarity, and simplicity of each item and rate it on a 4-point Likert scale ranging from 1 (the lowest) to 4 (the highest) (15). Polit et al. recommended a score of 0.78 or higher for accepting the CVI of an item as good content validity (16). 


\subsection{Statistical Analysis}

The data were analyzed with the SPSS software package (version 22). Cronbach's alpha was estimated to determine the internal consistency of the questionnaire. Intraclass Correlation Coefficient (ICC) was calculated to obtain the test-retest reliability coefficient. The ICC was calculated for total scores. ICC is classified as high (more than 0.90), moderate (between 0.75 and 0.90), and low (less than 0.75) (17). To assess the reliability, standard error measurement(SEM) and ICC were used. In this regard, ICC is a measure of reliability and represents the ability of a scale to distinguish between participants, while SEM is absolute reliability. SEM is described as SD $\sqrt{ }$ 1-ICC (18). Chi-square was used to compare the prevalence of insomnia in age groups of children.

\section{Results}

In this study, 209 children (103 boys and 106 girls) were evaluated. The mean age was $8.02 \pm 3.54$ years. Table 1 shows the descriptive characteristics of the subjects. Parents of children were asked to sign an informed consent form before starting the study.

\subsection{Internal Consistency}

Cronbach's alpha coefficient was 0.762 , and deletion of each item caused no significant change in alpha.

\subsection{Precision of Measurement}

The Standard Error of Measurement (SEM) values for the questionnaire are presented in Table 2. Accordingly, SEM was acceptable when the level of SEM was $<10 \%$ of the total mean score (19).

\subsection{Intraclass Correlation Coefficient}

To assess the reliability of Children's Sleep Comics, 30 children were retested after one week to calculate the intraclass correlation coefficient. Table 2 shows ICC coefficient values. The coefficient was 0.978 , which confirms the repeatability of this test.

The prevalence of insomnia symptoms among the children was $21 \%$, the results of which are presented in Table 1. There was no difference in the prevalence of insomnia between different age groups (P value $>0.05$ ).

\section{Discussion}

In this study, the psychometric properties (content validity and test-retest reliability) of the Persian version of Children's Sleep Comic were examined.

\begin{tabular}{|c|c|c|c|}
\hline $\operatorname{Age}(y)$ & & Frequency, No. (\%) & Insomnia, No. (\%) \\
\hline \multicolumn{4}{|l|}{$5-6$} \\
\hline & Male & $15(7.18)$ & $3(20.0)$ \\
\hline & Female & $12(5.74)$ & $2(16.7)$ \\
\hline \multicolumn{4}{|l|}{ 6-7 } \\
\hline & Male & $13(6.23)$ & $4(30.8)$ \\
\hline & Female & $15(7.18)$ & $4(26.7)$ \\
\hline \multicolumn{4}{|l|}{$7-8$} \\
\hline & Male & $15(7.18)$ & $6(40.0)$ \\
\hline & Female & $16(7.65)$ & $2(12.5)$ \\
\hline \multicolumn{4}{|l|}{ 8-9 } \\
\hline & Male & $16(7.65)$ & $1(6.3)$ \\
\hline & Female & $16(7.65)$ & $3(18.8)$ \\
\hline \multicolumn{4}{|l|}{ 9-10 } \\
\hline & Male & $16(7.65)$ & $3(18.8)$ \\
\hline & Female & $16(7.65)$ & $2(12.5)$ \\
\hline \multicolumn{4}{|l|}{ 10-11 } \\
\hline & Male & $15(7.18)$ & $4(26.7)$ \\
\hline & Female & $15(7.18)$ & $2(13.3)$ \\
\hline \multicolumn{4}{|l|}{ 11-12 } \\
\hline & Male & $13(6.23)$ & $3(23.1)$ \\
\hline & Female & $16(7.65)$ & $4(25.0)$ \\
\hline Total & & $209(100)$ & $44(21.0)$ \\
\hline
\end{tabular}

To achieve the study goals, the questionnaire was translated into Persian, and then its content validity was assessed. According to the opinion of experts, all items were essential and appropriate with acceptable CVR and CVI. Thus, the Persian version of the questionnaire is an acceptable tool for recognizing insomnia in children aged 5 to 11 years old. According to the results, test-retest reliability was 0.97, and Cronbach's alpha coefficient was 0.76 for the whole questionnaire. Therefore, the questionnaire has desirable reliability for Iranian children. Schwerdtle et al. in 2016 (12) reported Cronbach's alpha for one-on-one and group interviews, 0.83 and 0.80 , respectively, and high convergence validity for both instruments $(r=0.66)$. For the Children's Sleep Comic and the Children's Sleep Habits questionnaire, it was low to moderate $(r=0.32)$, which is consistent with previous studies and confirms that children score sleep is different from their parents.

One of the strengths of this study is that all the interviews were conducted one by one because, according to the test developer, this method is more valid and reliable (12). Our sampling was random and not voluntary, so it is un- 
Table 2. The Results of Intraclass Correlation Coefficient of the Questionnaire and SEM

\begin{tabular}{lcccc}
\hline Variable Results & Intraclass Correlation Coefficient & SD & SEM & P Value \\
\hline Children's Sleep Comic & 0.978 & 0.17 & 0.02 & $<0.01$
\end{tabular}

likely that only motivated parents and parents of children with sleep problems participated.

In the present study, the children under study were healthy, and no comparison was made with people having sleep problems. Content validity was used to calculate the validity, and mean content validity was also determined to be approximately $97 \%$ by the panel of experts.

These data suggest that one out of five young children and preadolescents (21\%) of the general population have insomnia symptoms. Schwerdlt et al. (2016) reported sleep disorders in children aged 5-11 years old close to $20 \%$, which is consistent with our study (12). Although sleep problems are said to decrease with the child's age, this was not the case in our samples. In fact, it is the pattern of sleep problems that changes with age. For example, preschool children resist going to bed and may wake up several times and need parental attention, such as sleeping with them. In school-age children, other factors such as night-time fears and worry and anxiety about daytime matters may be added to the previous issues (20). Another reason for the difference in results may be cultural issues that affect sleep patterns and problems (21).

According to the results, no sex differences were found in the entire sample or within age groups. It is said that after puberty, gender differences occur in insomnia (22). Sleep disorders in children are common but often remain undetected and are not managed. In assessing children's sleep disorders, parents are often asked about their children's sleep and sleep behavior. Because some of the child's sleep characteristics can be assessed by parents (e.g., bedtime, sleep status, or daytime disorder) and others can be better expressed by the child (e.g., anxiety or sleepwalking), both ratings are necessary. It is a childfriendly, self-report tool that assesses sleep and sleep behavior in young children who are unable to read and write. Likewise, it can build a good therapeutic relationship and pave the way for a successful intervention(12). Based on the results, children's sleep Comic is a reliable tool.

This study has some limitations; first, no comparison has been made with objective measurement; for example, polysomnography. The convergent and divergent validity of Children's Sleep Comic should be taken into consideration in future studies. Another limitation is that we did not determine the accuracy of the Persian version of Children Sleep Comic in diagnosing sleep disorders. Thus, children identified with sleep disorders should be confirmed by other tests.

\subsection{Conclusion}

The current study indicates that the Persian version of Children's Sleep Comic is a reliable and valid tool for screening insomnia among Iranian children aged 5-11 years old. This test is not sufficient for a diagnosis but can give precious information about the child's sleeping behavior and related issues. Children's Sleep Comic is also valuable to build a relationship between the child and therapist.

\section{Footnotes}

Authors' Contribution: Study concept and design: M. K., and M. V.; Acquisition of data: M.V.; Analysis and interpretation of data: S. T., and M. V.; Drafting the manuscript: M. V.; Critical revision of the manuscript for important intellectual content: M. K.; Statistical analysis: S. T, and M. V.; Administrative, technical, and material support: M. V; Study supervision: M. K.

Conflict of Interests: The authors declare no conflict of interest.

Ethical Approval: IR.SBMU.RETECH.REC.1398.393.

Funding/Support: This study was carried out with the financial support of Shahid Beheshti University of Medical Sciences, Tehran, Iran.

\section{References}

1. Lowry R, Eaton DK, Foti K, McKnight-Eily L, Perry G, Galuska DA. Association of Sleep Duration with Obesity among US High School Students. J Obes. 2012;2012:476914. doi: 10.1155/2012/476914. [PubMed: 22530111]. [PubMed Central: PMC3306918].

2. Fallahzadeh H, Etesam F, Asgarian FS. Validity and reliability related to the Persian version of the Children's Sleep Habits Questionnaire. Sleep Biol Rhythms. 2015;13(3):271-8. doi: 10.1111/sbr.12114.

3. Atrooz F, Salim S. Sleep deprivation, oxidative stress and inflammation. Adv Protein Chem Struct Biol. 2020;119:309-36. doi: 10.1016/bs.apcsb.2019.03.001. [PubMed: 31997771].

4. Hill CM, Everitt H. Assessment and initial management of suspected behavioural insomnia in pre-adolescent children. BMJ. 2018;363:k3797. doi:10.1136/bmj.k3797. [PubMed:30327294].

5. Calhoun SL, Fernandez-Mendoza J, Vgontzas AN, Mayes SD, Liao D, Bixler EO. Behavioral Profiles Associated with Objective Sleep Duration in Young Children with Insomnia Symptoms. J Abnorm Child Psychol. 2017;45(2):337-44. doi: 10.1007/s10802-016-0166-4. [PubMed: 27245765]. [PubMed Central: PMC7090378]. 
6. Simola P, Laitalainen E, Liukkonen K, Virkkula P, Kirjavainen T, Pitkaranta A, et al. Sleep disturbances in a community sample from preschool to school age. Child Care Health Dev. 2012;38(4):572-80. doi: 10.1111/j.1365-2214.2011.01288.x. [PubMed: 21790716].

7. Owens J. Classification and epidemiology of childhood sleep disorders. Prim Care. 2008;35(3):533-46. vii. doi: 10.1016/j.pop.2008.06.003. [PubMed: 18710669].

8. Chen WL, Chen JH. Consequences of inadequate sleep during the college years: Sleep deprivation, grade point average, and college graduation. Prev Med. 2019;124:23-8. doi:10.1016/j.ypmed.2019.04.017. [PubMed: 31034864].

9. Patrick Y, Lee A, Raha O, Pillai K, Gupta S, Sethi S, et al. Effects of sleep deprivation on cognitive and physical performance in university students. Sleep Biol Rhythms. 2017;15(3):217-25. doi: 10.1007/s41105017-0099-5. [PubMed: 28680341]. [PubMed Central: PMC5489575].

10. Badin E, Haddad C, Shatkin JP. Insomnia: the Sleeping Giant of Pediatric Public Health. Curr Psychiatry Rep. 2016;18(5):47. doi: 10.1007/s11920-016-0687-0. [PubMed: 26993792].

11. Schwerdtle B, Kanis J, Kahl L, Kübler A, Schlarb AA. Children's Sleep Comic: development of a new diagnostic tool for children with sleep disorders. Nat Sci Sleep. 2012;4:97-102. doi: 10.2147/NSS.S33127. [PubMed: 23620683]. [PubMed Central: PMC3630976].

12. Schwerdtle B, Kanis J, Kubler A, Schlarb AA. The Children's Sleep Comic: Psychometrics of a Self-rating Instrument for Childhood Insomnia. Child Psychiatry Hum Dev. 2016;47(1):53-63. doi:10.1007/s10578015-0542-2. [PubMed: 25783950].

13. Fayers PM, Machin D. Quality of Life: Assessment, Analysis and Interpretation. John Wiley \& Sons, Ltd; 2000. doi: 10.1002/0470846283.

14. Msyamboza KP, Ngwira B, Dzowela T, Mvula C, Kathyola D, Harries $\mathrm{AD}$, et al. The burden of selected chronic non-communicable diseases and their risk factors in Malawi: nationwide STEPS survey. PLoS One. 2011;6(5). e20316. doi: 10.1371/journal.pone.0020316. [PubMed:
21629735]. [PubMed Central: PMC3100352].

15. Waltz CF, Strickland OL, Lenz ER. Validity of measures. Measurement in nursing and health research. Springer publishing company; 2010.

16. Polit DF, Beck CT, Owen SV. Is the CVI an acceptable indicator of content validity? Appraisal and recommendations. Res Nurs Health. 2007;30(4):459-67. doi:10.1002/nur.20199. [PubMed: 17654487].

17. Van Gils A, Meyer S, Van Dijk M, Thijs L, Michielsen M, Lafosse C, et al. The Adult Assisting Hand Assessment Stroke: Psychometric Properties of an Observation-Based Bimanual Upper Limb Performance Measurement. Arch Phys Med Rehabil. 2018;99(12):2513-22. doi: 10.1016/j.apmr.2018.04.025. [PubMed: 29807004].

18. A. LIMIl E, Kalantari M, Nazeri AR, Akbarzade Baghban A. Test-retest \& Inter-rater Reliability of Persian Version of Pediatric Balance Scale in Children with Spastic Cerebral Palsy. Iran J Child Neurol. 2019;13(4):16371. [PubMed: 31645876]. [PubMed Central: PMC6789081].

19. Souza DC, Wegner F, Costa LCM, Chiavegato LD, Lunardi AC. Measurement properties of the Human Activity Profile questionnaire in hospitalized patients. Braz J Phys Ther. 2017;21(3):153-8. doi: 10.1016/j.bjpt.2017.03.011. [PubMed: 28473282]. [PubMed Central: PMC5537466].

20. Stores G. Aspects of sleep disorders in children and adolescents. Dialogues Clin Neurosci. 2009;11(1):81-90. [PubMed: 19432390]. [PubMed Central: PMC3181901].

21. Knutson KL. Sociodemographic and cultural determinants of sleep deficiency: implications for cardiometabolic disease risk. Soc Sci Med. 2013;79:7-15. doi: 10.1016/j.socscimed.2012.05.002. [PubMed: 22682665]. [PubMed Central: PMC3443287].

22. Zhang J, Chan NY, Lam SP, Li SX, Liu Y, Chan JW, et al. Emergence of Sex Differences in Insomnia Symptoms in Adolescents: A Large-Scale School-Based Study. Sleep. 2016;39(8):1563-70. doi: 10.5665/sleep.6022. [PubMed: 27091537]. [PubMed Central: PMC4945316]. 